\title{
A randomised controlled trial examining the efficacy of smoking-related response inhibition training in smokers: a study protocol
}

Petra K. Staiger ${ }^{1,2^{*}}$ (D) Melissa J. Hayden ${ }^{1,2,3}$, Karen Guo ${ }^{1}$, Laura K. Hughes ${ }^{1}$, Jason Bos ${ }^{1}$ and Natalia S. Lawrence ${ }^{4}$

\begin{abstract}
Background: Smoking is one of the leading preventable causes of illness and premature death worldwide. Despite a variety of effective treatments, relapse rates remain high, and novel, innovative interventions are needed in order to reduce the global prevalence of smoking. Research has indicated that deficits in the ability to inhibit a response (referred to as response inhibition) is a predictor of relapse and subsequently, targeting this potentially modifiable risk factor may lead to improvements in smoking outcomes. Indeed, in recent years, stimulus-specific response inhibition training has emerged as a potentially efficacious intervention to reduce unwanted/unhealthy behaviours such as alcohol and unhealthy food consumption. As such, the present trial is the first to evaluate the real-world efficacy of response inhibition smoking training (INST) in a sample of adult heavy smokers.
\end{abstract}

Methods/design: This randomised controlled trial will recruit nicotine dependent smokers aged between 18 and 60 using social media and advertisements in Victoria, Australia. The sample target was 150 to account for drop out and non-adherence. Once informed consent has been obtained, participants complete a range of baseline measures during a face to face interview. Participants are randomly allocated to one of two online training conditions: an intervention training group (INST), which requires participants to exercise response inhibition towards smoking-related stimuli; or an active control group, which requires participants to exercise response inhibition towards household items and does not include any smoking-related stimuli. They complete the first training session during the interview to ensure the training protocol is clear. Both groups are instructed to complete a further 13 training sessions (1 per day) at home on their computer and follow-up phone calls will be conducted at three time points: post-intervention, one-month and three months. The primary outcomes are: a) rates of smoking cessation and; b) reduction in the quantity of average daily smoking at post-intervention, one and three months follow-up.

Discussion: There is a pressing need to develop novel and innovative smoking interventions. If proven to be effective, INST could make a highly cost-effective contribution to improvements in smoking intervention outcomes.

Trial registration: The trial was prospectively registered with the Australian New Zealand Clinical Trials Registry 17th February 2017. Trial ID: ACTRN12617000252314.

Keywords: Smoking cessation, Response inhibition, Inhibitory control, Cognitive training, Devaluation, eHealth, Craving, Intervention

\footnotetext{
* Correspondence: petra.staiger@deakin.edu.au

${ }^{1}$ School of Psychology, Deakin University, Geelong, VIC 3220, Australia

${ }^{2}$ Centre for Drug Use, Addictive and Anti-social behaviour Research

(CEDAAR), Deakin University, Locked Bag 20000, Geelong, VIC 3220, Australia

Full list of author information is available at the end of the article
}

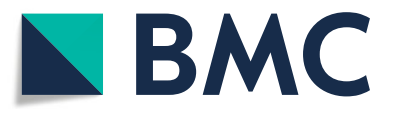

(c) The Author(s). 2018 Open Access This article is distributed under the terms of the Creative Commons Attribution 4.0 International License (http://creativecommons.org/licenses/by/4.0/), which permits unrestricted use, distribution, and

reproduction in any medium, provided you give appropriate credit to the original author(s) and the source, provide a link to the Creative Commons license, and indicate if changes were made. The Creative Commons Public Domain Dedication waiver (http://creativecommons.org/publicdomain/zero/1.0/) applies to the data made available in this article, unless otherwise stated. 


\section{Background}

Tobacco smoking is one of the leading preventable causes of illness and premature death worldwide. It is the second largest contributor to the burden of disease globally, with 134.2 million years lost to disability, illness and premature death [1]. In 2013, 6.1 million people died globally as a result of tobacco use [1], and, if trends persist, this number will exceed 8 million by the year 2030 [2]. Furthermore, tobacco use has been found to cost the global economy more than US\$1 trillion each year in healthcare expenditures and lost productivity [3]. As smoking remains at unacceptable levels across the world [4, 5], examinations of effective and accessible smoking cessation treatments are crucial in reducing the global burden of smoking on public health.

Currently, pharmacological and psychosocial interventions have the most support as efficacious treatments for the cessation of smoking [6-10]. However, despite the positive outcomes associated with these interventions, most smokers do not seek formal treatment to reduce smoking [11] and existing treatments can entail several limitations. First, smokers have reported concerns regarding adverse side-effects of pharmacological treatments which have impacted treatment uptake and long-term adherence [12-14]. Second, the long-term cost of pharmacological and psychosocial interventions, which can be more expensive than cigarettes themselves, often prohibit individuals from accessing smoking cessation treatments. This is particularly relevant given that: 1) the financial costs of tobacco are one of the primary reasons underpinning quit intentions and attempts [4, $15,16]$ and; 2) the incidence of smoking is increasing most rapidly in developing nations who have the lowest levels of disposable income [5]. Therefore, there is a critical need for accessible and cost-efficient interventions for smoking cessation. Third, relapse rates remain consistently high following treatment [17] and, the vast majority will relapse within five to 10 days of treatment cessation $[18,19]$. Thus, a substantial proportion of individuals attempting to quit smoking fail to achieve long-term abstinence, inviting the question: what modifiable risk factors for smoking relapse may be targeted to increase abstinence rates or at the very least result in reduction of level of smoking?

Previous research indicates that deficits in response inhibition are a strong predictor of relapse for smokers following a quit attempt [20, 21]. Research suggests that recently abstinent smokers experience heightened difficulties with response inhibition [22, 23], indicating that targeting this may assist in preventing relapse. Importantly, a meta-analysis [23] supports evidence showing that individuals dependent on substances such as cocaine and alcohol may experience deficits in response inhibition. Furthermore, Yin and colleagues [24] found that a group of smokers reported response inhibition deficits on the GNG task. Taken together this provides some evidence that smokers may experience difficulties with response inhibition. Of significance is that individuals who reported higher nicotine dependence experienced greater deficits in response inhibition than those of lower use or dependence [25, 26]. Given that heavier smokers find it more difficult to quit [27, 28], response inhibition deficits may be an effective target for treatment in these individuals.

Indeed, response inhibition training interventions utilising tasks such as the go/no-go (GNG) task and stop signal task (SST) focus on training successful inhibition of a habitual or pre-potent response by pairing pictorial cues of the targeted behaviour with stop signals or no/go cues [29]. The GNG task targets automatic bottom-up response inhibition (or action restraint) by consistently pairing no-go cues with the target stimuli [30, 31], while the SST targets top-down inhibitory control (or action cancellation) as stop signals occur after an initiated response and are mapped only to a proportion of target stimuli [32]. These tasks have recently been examined to reduce alcohol and food intake, yielding efficacious results [33]. For example, Houben, Havermans, Nederkoorn, and Jansen [34] randomly assigned 57 heavy alcohol drinkers to receive one of two training conditions: a beer/no-go condition, where alcohol-related stimuli were consistently paired with a stopping response, or a beer/go condition, where participants always responded to alcohol-related stimuli. Compared to participants in the beer/go condition, those who were trained to inhibit their response towards alcohol-related stimuli (beer/no-go) reported significantly less alcohol intake. Similar findings were reported by Jones and Field [35]. In their study, following motor inhibition training utilising a modified SST, heavy social drinkers were found to consume significantly less alcohol in a subsequent ad libitum taste test.

More recently Lawrence et al. [36] implemented an internet-delivered response inhibition training intervention for food among 83 overweight and obese adult participants. Participants were randomly allocated to receive four 10-min training sessions completed online. In the intervention group, high-calorie foods were consistently paired with no-go signals and in the control group, non-food stimuli were consistently paired with no-go signals. At one-week follow-up, participants in the food no-go condition consumed significantly less food, showed significant weight loss, and had decreased positive evaluations towards high calorie foods compared to controls. At 6 month follow-up, participants in the intervention group displayed significantly higher average weight loss $(2.21 \mathrm{~kg})$ compared to controls $(0.36 \mathrm{~kg})$. These findings are consistent with a previous trial [37] 
that compared two interventions for losing weight: an implementation intention intervention that instructed participants to plan reminders for dieting and a response inhibition intervention that paired no-go responses with food-related stimuli. Findings indicated that participants who completed only the response inhibition training reported significant weight loss after four training sessions. Together, these results indicate that response inhibition training can be effectively delivered online, promoting greater accessibility and cost-efficiency of these types of interventions.

Two meta-analyses have found that inhibitory control training resulted in an overall significant effect (albeit a small effect size), with GNG training yielding larger (medium) effect sizes than SST training [29, 33]. According to the Behaviour Stimulus Interaction (BSI) theory [38] behavioural changes induced by the GNG training are mediated by changes in evaluations of the stimuli used in the task. That is, positively regarded stimuli will become associated with negative affect as a result of consistently being paired with no-go cues. This is thought to devalue the stimuli and minimise the likelihood of approach behaviours occurring towards the stimuli in real life. This theory has been supported by evidence in studies targeting alcohol consumption that suggest a mediating effect of changes in implicit attitudes on alcohol intake [34, 39]. In the food domain, there is evidence of devaluation of trained no-go food stimuli as assessed by visual analogue scales [36, 40, 41]. Another proposed mechanism of response inhibition training is the automatic inhibition hypothesis (AIH) [32], which posits that automatic response inhibition can develop over practice if stimuli are consistently associated with stopping $[42,43]$. These two potential mediating hypotheses will be investigated in this trial.

In summary, given that significant effects of the GNG task were found despite the use of non-clinical samples, it was expected that these interventions would be particularly effective with smokers as smoking receives the most frequent reinforcement compared with other dependent populations, with multiple smoking sessions each day. Furthermore, we hypothesise that it will be particularly beneficial for heavy smokers who report the greatest difficulty with impulse control [4]. This is suggested by findings that stronger nicotine dependence is associated with poorer inhibitory control [44]. Thus, this is the first study to use the GNG task in a sample of individuals who have a Tobacco Use Disorder according to DSM-5 criteria and who wish to quit/reduce smoking.

As previous studies have found response inhibition training to be effective even when administered over the internet [36, 37], this study delivered the training paradigm online. This enabled the intervention to be accessible, convenient and cost-efficient for individuals and further contribute to reducing the burden on other treatment services and resources. The study is a randomised controlled trial (RCT) examining the efficacy of response inhibition training in reducing smoking in heavy dependent smokers. It is implemented in accordance with CONSORT guidelines, and involves collecting follow-up data from participants at 1 month and 3 months post-intervention.

\section{Primary hypotheses}

1. Smokers who received smoking-related response inhibition training (INST program) would report significantly higher cessation rates compared to those in the active control condition at the end of the intervention, 1 month and 3 months post-intervention.

2. Smokers who received smoking-related response inhibition training (INST program) would report significantly less cigarette consumption compared to smokers in the active control condition at the end of the intervention, 1 month and 3 months post-intervention.

\section{Secondary hypotheses}

1 Smokers who received smoking-related response inhibition training (INST program) would report significantly less craving for cigarettes compared to smokers in the active control condition at the end of the intervention and 1 month and 3 months post-intervention.

2 Smokers who received smoking-related response inhibition training (INST program) would report significantly lower levels of nicotine dependence compared to smokers in the active control condition at the end of the intervention and 1 month and 3 months post-intervention.

\section{Predictor/moderator hypotheses}

1. Individuals reporting high levels of impulsivity would report significantly improved outcomes from the intervention training compared to those with lower levels of impulsivity

2. Individuals who completed a greater number of sessions (i.e., dose) would report significantly improved outcomes from the intervention training compared to those who completed less sessions.

\section{Mediator hypotheses}

1. The effects of INST training on level of smoking would be mediated by devaluation of smoking 
stimuli as measured by a devaluation of smoking images task.

2. The effects of INST training on level of smoking would be mediated by an independent measure of response inhibition (SST).

The following exploratory question was proposed:

1. Do smokers who receive smoking-related response inhibition training (INST program) report significantly higher levels of self-confidence and motivation to quit smoking compared to smokers in the active control condition at the end of the intervention, 1 month and 3 months post-intervention.

\section{Methods/design}

\section{Design}

This is a 2-group parallel-block double-blind randomised controlled trial testing the efficacy of an intervention compared to an active control training. The intervention training is a smoking version of the food GNG training task in Lawrence et al. [36]. The active control training is similar to the control training in Lawrence et al. [36], with no-go training to household items. The Deakin University Human Research Ethics Committee (DUHREC) reviewed and approved all relevant study materials (Project ID: 2015-298). The trial was registered with the Australian New Zealand Clinical Trials Registry (Trial ID: ACTRN12617000252314; see Additional file 1: Table S1 for items from the World Health Organisation Data Set as per Spirit Guidelines). No study protocol amendments were made once the trial commenced and this protocol was originally submitted to this journal 1 November 2017.

\section{Procedure}

The following sections describe the study procedure. See Table 1 for an overview.

\section{Initial screening}

Participants were adult smokers aged between 18 and 60 years, recruited through social media and advertisements in Victoria, Australia who had a desire to quit smoking.

\section{Inclusion criteria}

- Aged between 18 and 60 years.

- Smoke, on average, a minimum of 10 cigarettes per day for the last 12 months.

- Meet criteria for moderate or above Tobacco Use Disorder defined by the DSM-5 [45].

- Be motivated to make a quit attempt during the training stage of the intervention.

- Completed at least Year 9 (or equivalent) schooling.
- Have computer and internet access during the intervention phase of the study.

\section{Exclusion criteria}

- Primarily uses electronic cigarettes on a daily basis.

- Non-smoking period of 2 weeks or more in the past 3 months.

- Currently using anti-craving medication.

- Using nicotine-replacement therapy during the intervention period.

- Self-reported problematic alcohol or drug(s) use other than tobacco.

- Reported a traumatic or acquired brain injury or a loss of consciousness for more than $30 \mathrm{~min}$.

- Reported current use of psychotropic medication such as anti-depressant, anti-psychotic and/or anxiolytic medication.

Interested participants were invited to contact the research team via email. They were screened over the phone/online to determine their eligibility. Participants who met the inclusion criteria were invited to participate in the study and attended a face to face interview in order to sign the consent form, collect baseline measures and participate in the first online training session.

\section{Baseline assessment (T1)}

At the beginning of the baseline interview session, participants read the plain language statement and if in agreement signed the consent form. They were requested to report any adverse events or consequences which will be reported in the flow chart of the primary outcomes paper. They were informed that they were able to withdraw from the study at any time. They were asked to indicate whether they would like to receive a summary of the trial findings following completion of data analyses. Participants were informed that they would receive one of two brain training tasks as the aim of the study was to investigate which one was more effective. While they were informed that the task incorporated a "variety of visual images", the types of images were not specified to prevent participants from identifying if they were in the control group and hence we propose that participants were likely blind to the nature of the intervention and whether they were randomised to an active condition.

Participants completed a battery of questionnaires (outlined in Table 1), and completed ratings of their craving, motivation and self-efficacy. Following the completion of the questionnaires, participants completed ratings of stimulus evaluation test and a smoking stop signal task (SST), an independent measure of response inhibition separate to the response inhibition training. 
Table 1 SPIRIT Flow Diagram of the schedule for participants and data collection for the INST study

\begin{tabular}{|c|c|c|c|c|c|c|c|}
\hline & \multirow[b]{3}{*}{ Enrolment } & \multicolumn{6}{|l|}{ STUDY PERIOD } \\
\hline & & \multirow[b]{2}{*}{$\begin{array}{l}\text { Baseline (T1) } \\
\text { and Allocation }\end{array}$} & \multirow[b]{2}{*}{$\begin{array}{l}\text { Training } \\
\text { Period }\end{array}$} & \multicolumn{3}{|l|}{ Follow-Up Period } & \multirow[b]{2}{*}{ Close-out } \\
\hline & & & & Post-Intervention (T2) & $\begin{array}{l}\text { 1-Month Post- } \\
\text { Intervention (T3) }\end{array}$ & $\begin{array}{l}\text { 3-Months Post- } \\
\text { Intervention (T4) }\end{array}$ & \\
\hline TIMEPOINT & $-t_{1}$ & $t_{0}-t_{7}$ & $t_{2}-t_{14}$ & $t_{15}$ & $t_{45}$ & $t_{105}$ & $t_{x}$ \\
\hline \multicolumn{8}{|l|}{ ENROLMENT: } \\
\hline Eligibility screen & $x$ & & & & & & \\
\hline Informed consent & $x$ & $x$ & & & & & \\
\hline Obtain Contact Information & $x$ & $x$ & & & & & \\
\hline Allocation & & $x$ & & & & & \\
\hline \multicolumn{8}{|l|}{ INTERVENTIONS: } \\
\hline INST intervention & & $x$ & $x$ & & & & \\
\hline Control training & & $x$ & $x$ & & & & \\
\hline \multicolumn{8}{|l|}{ ASSESSMENTS: } \\
\hline Demographic Questions & & $x$ & & & & & \\
\hline$T L F B$ & & $x$ & & $x$ & $x$ & $x$ & \\
\hline FTND & & $x$ & & $x$ & $x$ & $x$ & \\
\hline DASS & & $x$ & & $x$ & $x$ & $x$ & \\
\hline AUDIT & & $x$ & & & & $x$ & \\
\hline$B I S-11$ & & $x$ & & $x$ & $x$ & $x$ & \\
\hline Stimulus Evaluation Test & & $x$ & & $x$ & $x$ & $x$ & \\
\hline SST & & $x$ & & $x$ & & $x$ & \\
\hline Craving Rating & & $x$ & $x$ & $x$ & $x$ & $x$ & \\
\hline Motivation Rating & & $x$ & & $x$ & $x$ & $x$ & \\
\hline Confidence Rating & & $x$ & & $x$ & $x$ & $x$ & \\
\hline Time of last cigarette & & $x$ & $x$ & & & & \\
\hline CLOSE-OUT: & & & & & & & $x$ \\
\hline Data-analysis & & & & & & & $x$ \\
\hline Debriefing of participants & & & & & & & $x$ \\
\hline $\begin{array}{l}\text { Documentation and Dissemination } \\
\text { of Findings }\end{array}$ & & & & & & & $x$ \\
\hline
\end{tabular}

t refers to days (from $\mathrm{t}_{1}$ onwards). TLFB Timeline Follow-Back interview, FTND Fagerström Test of Nicotine Dependence, DASS Depression Anxiety and Stress Scale, AUDIT Alcohol Use Disorders Identification Test, BIS-11 Barrett Impulsiveness Scale, SST Stop Signal Task

\section{Randomisation}

Immediately following the completion of the baseline assessment, participants began the online training task. Participants were automatically randomised to either the intervention or the control training task via a pre-computed randomisation procedure. A permuted block randomisation procedure was utilised [46] whereby participants were allocated to the intervention or control group through the use of a randomly generated number. The permuted blocks were organised in groups of ten, the details of which were not known by investigators involved with the administration of the trial. The use of the permuted block randomisation process ensures that intervention group numbers will be balanced at the end of each block and is thus the recommended process in studies with smaller samples.

Upon finishing this task participants were instructed to complete the online training task once per day for the next 13 days, totalling 14 sessions. They were asked to rate their smoking craving level before and after each training session. Twice per week, participants were sent text reminders to complete the training. All data from the online training task and outcome measures were securely stored on the Deakin University server and linked to an anonymous participant ID number such that only de-identified data were available to researchers. The data was checked for training task performance accuracy and 
participant adherence to the training protocol by a research assistant who was independent from investigators and not involved in data collection or analyses.

\section{Inhibition training task}

The intervention is an online GNG training task as developed by Lawrence et al. [36], modified to incorporate images of smoking. The task included nine smoking images (or household items in the control group), nine relaxation images (or household items in the control group) and 18 neutral filler images presented on the left or right of the computer screen (see Fig. 1). Each image was presented for $1250 \mathrm{~ms}$ followed by a $1250 \mathrm{~ms}$ inter-stimulus interval. Participants were instructed to indicate whether the image is located to the left or the right of the screen using the keys "C" and " $\mathrm{M}$ " respectively on their keyboard. On half of the trials, the frame around the picture was bolded and the participants were required to not respond (no-go trials). On the other half of the trials the frame was not bolded (go trials) and the participant were required to respond as quickly as possible. During each training session participants completed 6 training blocks, with each of the 36 images presented once per block. At the end of each block, participants were provided with feedback on their accuracy and mean correct go reaction time and will be encouraged to continue trying to beat their own score. Each training session will last for approximately $10 \mathrm{~min}$. Participants were asked to complete the training at home in a quiet place and preferably, when they experienced cravings for a cigarette.

\section{Intervention group}

The intervention consisted of nine smoking-related images, nine relaxing images.

(i.e. depicting relaxing/enjoyable activities), and 18 neutral filler pictures (e.g. clothing). For the intervention

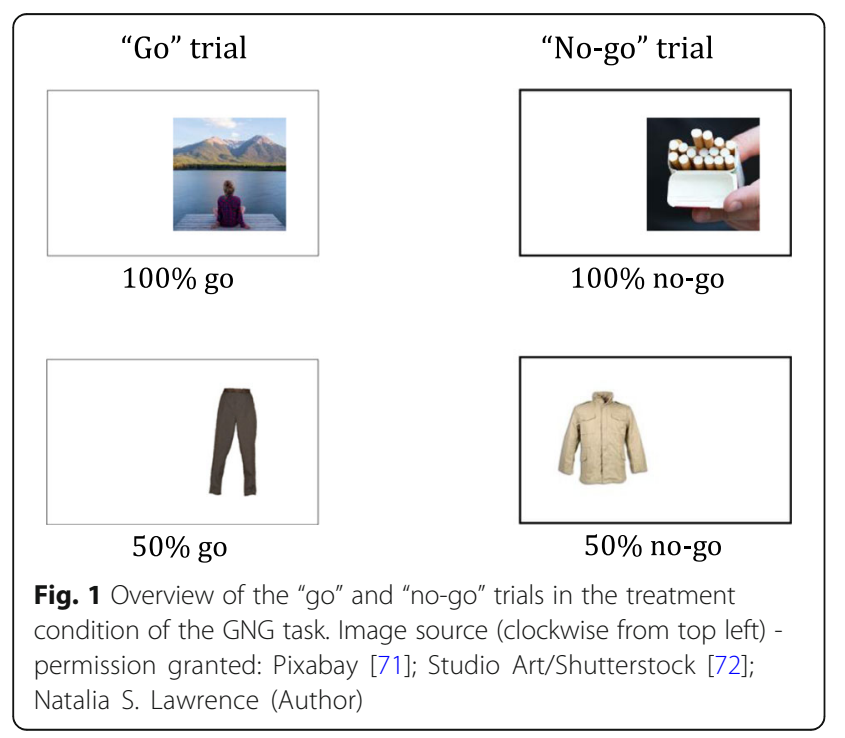

group, the smoking-related pictures were always "no-go" trials and the non-smoking pictures were always "go" trials. The neutral pictures were equally "go" and "no-go" trials (see Fig. 1). The neutral filler pictures were incorporated to prevent participants from easily identifying the associative rules of the task and to ensure the task remains challenging and engaging.

\section{Control group}

In the control group, participants complete a similar task to the smoking intervention group except that randomly presented 18 images of household objects replace the 18 smoking and relaxation images. The household images were presented equally as "go" and "nogo" trials.

\section{Post-intervention (T2)}

At the completion of the two-week intervention period, participants are contacted via phone by a researcher naïve to the group randomisation (i.e. a different researcher to the one who conducted the baseline interview). They receive a text message reminder $24-\mathrm{h}$ prior to confirm the time of the phone call. During these phone interviews, participants are asked to provide details about their use of cigarettes and nicotine replacement therapies or anti-craving medications over the previous 2 weeks. At the conclusion of this interview, participants are emailed a link to complete the same battery of questionnaires, ratings of their craving, motivation and self-efficacy and SST (completed last) as completed at baseline (T1).

\section{One-month and three-months follow-up (T3 and T4)}

Follow-up at 1 month (T3) and 3 months (T4) are conducted in the same manner as T2. The two follow-up time points are identical with the exception that the SST was not completed at T3 only in T4 to reduce participant burden. At the completion of each time point, participants were mailed a $\$ 20$ gift card. At the conclusion of the data collection period, participants in the control group are offered the opportunity to complete the smoking-related response inhibition training.

\section{Measures}

This study used information from a face-to-face interview session (T1) and phone interviews (T2, T3 and T4), in addition to self-report questionnaires, a cognitive task and a stimulus evaluation test. A list of measures used at each assessment point is provided in Table 1. Demographic information, such as age, gender, socioeconomic status and number of years of smoking, were collected at baseline.

\section{Researcher-administered measures}

Timeline Follow-Back (TLFB) [47, 48]

The TLFB is a calendar-based assessment of daily cigarette use for periods of time ranging from 1 to 
12 months prior to assessment. Initially developed to assess alcohol consumption, the TLFB has since been utilised to assess a variety of substance use inclusive of cigarette use [47]. Memory aids are used to enhance recall of certain time-periods in order to retrospectively estimate number of cigarettes used for each date. The cigarette TLFB has shown high test-retest reliability and temporal stability across both clinical and non-clinical participants [47].

\section{Self-report measures}

\section{Fagerström Test for Nicotine Dependence (FTND) [49]}

The FTND is a six-item self-report questionnaire of nicotine dependence. Dichotomous items (yes or no) are scored as $0-1$, and options for categorical items are scored $0-3$. The FTND has a maximum score of 10 , with higher scores indicating greater nicotine dependence. The FTND demonstrates moderate internal consistency $(\alpha=.61)$ and has been validated in smokers from the general population [48] and in a clinical sample [50].

\section{Craving for cigarettes}

A one-item question utilising a $100 \mathrm{~mm}$ slider scale measures craving from "not at all" to "extremely". Participants respond to the question "How much are you currently craving a cigarette". A slider bar is presented at the left end of the scale and participants will click and drag the bar along the scale to indicate their response. It has been found that a single measure of craving is just as reliable and sensitive as self-report questionnaires for measuring craving for smoking $[51,52]$. Slider scales are considered to be an engaging type of interface [53] and are regarded as a psychometrically acceptable measurement [54].

\section{Depression, Anxiety and Stress Scale (DASS-21) [55]}

The DASS-21 is a 21-item measure consisting of three subscales: depression, anxiety, and stress. Participants are asked to use a four-point Likert scale to rate the extent to which they have experienced the state described over the past week. The DASS has excellent internal consistency for the total scale $(\alpha=.97)$, and each subscale $($ Depression $=.96$; Anxiety $=.92$; Stress $=.95$ ) has high test-retest reliability and acceptable construct and convergent validity [56].

\section{Alcohol Use Disorders Identification Test (AUDIT) [57]}

The AUDIT is a 10-item measure of alcohol problems. Questions relate to frequency and quantity of consumption, and alcohol-related problems. Participants are asked to rate items from 0 to 4 and can receive a maximum possible score of 40 , with higher scores indicative of more hazardous drinking, AUDIT is highly reliable and valid for use across a range of populations [58].

\section{Barratt Impulsiveness Scale (BIS-11) [59]}

The BIS-11 is a 30-item questionnaire assessing trait impulsivity. Each item is scored on a four-point Likert scale that ranges from "rarely/never" to "almost always". Scores are summed to yield an overall total score ranging from 30 to 120 , with higher scores indicating greater trait impulsivity. The BIS-11 also provides scores on three subscales: attentional impulsiveness, motor impulsiveness, and non-planning impulsiveness. The BIS-11 is widely used in research and clinical contexts and has been shown to demonstrate good reliability $[59,60]$.

\section{Ratings of motivation and self-efficacy}

Participants are asked to rate their motivation ("currently, how motivated are you to reduce or quit smoking?") and self-efficacy ("currently, how confident are you in your ability to quit or reduce smoking?") on slider scales. The scale is a $100 \mathrm{~mm}$ line with the left anchor labelled "not at all" and the right anchor labelled "extremely". Similar to the craving slider scale, participants indicate their response by clicking and dragging the slider bar along the scale.

\section{Stimulus evaluation test (ratings of likeability of smoking and relaxing images)}

Slider scales are used for the likeability ratings of the smoking and relaxing images used in the inhibition training task (INST). Participants are presented with the question, "how much would you like to do this activity right now?" and rate the images from "not at all" to "extremely". The slider bar is presented in the middle of the scale and participants click and drag the slider bar to indicate their response.

\section{Cognitive task}

Stop Signal Task (SST) [32, 61, 62]

A smoking-specific version of the SST [30, 32, 61-63] is utilised. The SST contains images of smoking-related stimuli that are different images from those used in the intervention task. Participants are presented with a fixation cross in the centre of a screen on a white background for $500 \mathrm{~ms}$. A smoking-related image (go-stimulus) then appears for $1000 \mathrm{~ms}$, followed by a blank white screen for $1000 \mathrm{~ms}$ (inter-stimulus interval). The $16 \mathrm{im}$ ages used in the SST are comprised of 8 pairs of images, where one image of the pair is a cigarette pointing to the left, and the second image is its mirror image pointing to the right. As such, the presentation of stimuli pointing left or right will be equally balanced. Each of the 16 images is presented a total of 12 times.

Participants are instructed to indicate whether the cigarette is pointing left or right by pressing the computer keys "C" or " $\mathrm{M}$ " respectively (Fig. 2). The stop signal is a pair of red lines across the image and will appear 


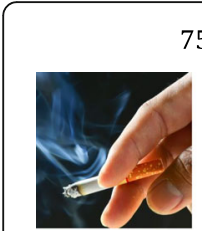

left
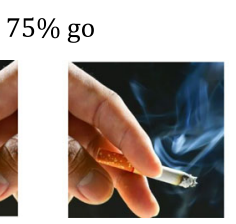

right

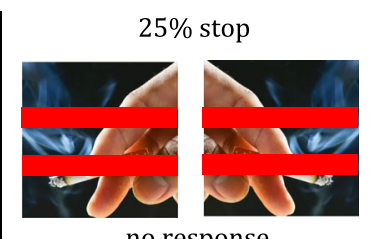

no response
Fig. 2 Overview of the "go" and "stop" trials in the Stop Signal Task illustrating correct responding. Image source - permission granted: Khamiranon D/Shutterstock [73]

on $25 \%$ of trials. It appears at a short delay (Stop Signal Delay or SSD) after the onset of the go stimulus and stays on screen until the inter-stimulus interval. Participants are instructed to respond as quickly as possible but to not respond when the red lines appear. This delay between the onset of the go signal and the stop signal begins at $250 \mathrm{~ms}$ on the first stop trial, and then adjusted by $50 \mathrm{~ms}$ in a staircase manner. Successful inhibition on stop trials results in the SSD increasing for the next stop trial, while unsuccessful inhibition, where the participant responds on a stop trial, will shorten this delay by $50 \mathrm{~ms}$. The SST consists of one practice block of 10 trials followed by the experimental block of 192 trials. The SSD will be used to calculate the stop signal reaction time (SSRT) as a measure of response inhibition and the reaction time on go trials will be a measure of behavioural impulsivity [62].

\section{Analysis plan}

All participants will be included in the intent-to-treat analyses for the primary and secondary hypotheses. If relevant, per protocol analysis will include those who complete at least four sessions of the training (as per Lawrence et al. [36]) and achieve a training accuracy of at least 70\%. SST data will be included for those who yield an accuracy of $40-60 \%$ on stop trials and at least $70 \%$ on go trials. Prior to analyses, all variables will be examined through IBM Statistical Package for Social Sciences (SPSS Version 25) for accuracy of data entry, missing values and fit between their distributions and the assumptions of multivariate analysis. Any violations will be addressed as per standard protocols [64].

Missing data will be managed using SPSS. First, a missing value analysis will be conducted to determine the percentage and pattern of missing data. If missing data are found to relate to a measured participant variable, those variables will be included as covariates in the analyses. If appropriate, multiple imputation will be used to replace missing values and the imputation model will include baseline covariates and outcome data. Missing data will be imputed using the Markov Chain Monte Carlo method or the Monotone method, contingent upon the pattern of missing data. A minimum of five imputed datasets will be produced [65]; however, depending on the percentage of missing data, a minimum of 20 imputed datasets may be required [66]. Wherever possible, results from the complete case analysis will be compared with results based on imputed data. If there are important differences, explanations will be offered.

The primary and secondary hypotheses will be analysed using separate mixed-design ANOVAs and a Chi-square analysis for the binary outcome. Depending on the rate of smoking abstinence at follow up the outcome variable will be calculated as either binary (smoking abstinence: yes/no) or percent days abstinence if Chi-square analysis is contraindicated due to low numbers in each cell. For all other mixed design ANOVAs group (i.e., intervention or control) will be included as the between-subjects factor and time (survey time points) is the within-subjects factor. For the smoking reduction primary hypothesis, the repeated-measures factor will be the average number of cigarettes smoked per day at each timepoint (i.e., baseline, post-intervention, 1 month and 3 months post intervention). For the secondary hypotheses, the repeated-measures factor will be craving or nicotine dependence at each timepoint. The predictor hypotheses will be examined using separate moderated regression analyses, with group as the predictor variable, impulsivity and dose as the moderator variable and change in smoking as the dependent variable.

The two mediation hypotheses will be analysed utilising a linear mixed model approach to examine whether the effects of INST training on level of smoking will be mediated by devaluation of smoking stimuli or an independent measure of response inhibition (SSRT).

The exploratory questions related to self-confidence and motivation will be examined using a separate mixed-design ANOVA, with group included as the between subjects factor and self-confidence and motivation at each timepoint included as the repeated-measures factor.

Repeated measures ANOVAs will be performed on "go" reaction times and "no-go" accuracy to examine stimulus-specific learning effects (100\% stimuli vs. $50 \%$ stimuli) over time (first vs. fourth training session as per the analysis by Lawrence and colleagues to allow comparability). Evidence of learning across the two time points will be indicated by faster reaction time on $100 \%$ go stimuli and fewer errors on 100\% no-go stimuli. Any further exploratory analyses will be labelled as such in the publication.

\section{Power analysis}

As previous ICT studies have not targeted abstinence the current study was powered on smoking reduction based on Lawrence et al., [36] weight reduction ICT outcome data. Power analysis conducted via G*power indicated that an overall sample size of 92 is required to detect a medium effect size (approximately .50 cohen's d based on Lawrence et al.) at the .05 alpha level using linear 
techniques (power $=.80$ ). Given that it is expected that approximately $25 \%$ will be lost to follow-up and up to $30 \%$ would not complete a minimum of 4 sessions, the target of the current study was set at 150 at the time of trial registration. However, estimated target sample may be amended if attrition is better than expected.

\section{Discussion}

Despite a decline in smoking rates prevalence of tobacco smoking still remains unacceptably high. Many pharmacological and psychosocial interventions for smoking are restricted in accessibility due to barriers such as cost and easy access. This trial has been designed to deliver internet-based response inhibition training in order to offer a simple, low-cost, and easily accessible smoking cessation/reduction intervention. As such, even small effect sizes of the intervention may translate to cumulatively large gains to public health. The current study protocol has been designed to examine the efficacy of response inhibition training to assist dependent smokers to cease or reduce cigarette use.

The intervention has several strengths regarding its timing, delivery and content. Firstly, the intervention maximises the use of being an internet-based program, which capitalises on the ability to have a wide reach within the community at a relatively low cost. This ensures that the intervention is both convenient and highly accessible given that the majority of the population have access to a computer. Secondly, while there is currently limited evidence to suggest that training response inhibition to smoking cues reduces cigarette use or craving [67], previous studies suggest that online response inhibition training to energy-dense food images helps individuals reduce their food intake, weight and food liking [36, 37, 68]. Thirdly, it has been suggested [69] that the best test of stimulus-specific response inhibition training is to use real-world studies that adopt a mixed between- and within-subjects design with repeated-measures (pre to post-intervention). This allows changes from baseline to be computed for meaningful/ecologically valid outcome measures.

While the usual process in translational research is to conduct "proof of concept" studies in the lab before attempting trials in the real-world, we decided to proceed straight to a real-world RCT of smoking-related response inhibition training based on the promising findings in eating behaviour and weight change. This is because laboratory studies can only measure acute training effects that may have little application or predictive value for real-world effects, and because laboratory studies typically adopt a single-session, between-groups design with the dependent variable often being measured only once post-training. This design is limited by confounds such as only one group being exposed to smoking cues during training. Furthermore, if the training relies on changing stimulus-response associations [43], it may be more effective at inducing behavioural change when conducted in real-world contexts associated with smoking (such as the home or workplace) than when conducted in a neutral laboratory setting.

A number of limitations need to be considered. Ongoing studies need to include an objective measure of nicotine use and larger samples in order to adequately power mediation analyses.

\section{Considerations for future research}

If this trial suggests positive effects of smoking-related response inhibition training, future research will need to determine how to optimise outcomes for smokers. Furthermore, research could include examining which aspects of the intervention will produce particular effects. This is both in terms of the training schedule (frequency, duration, timing and location of the training) and the mode of delivery (e.g. online vs. smartphone delivery). Smartphone apps and digital interventions to assist with smoking cessation are very popular but are largely lacking in evidence [70]. This RCT aims to contribute to the evidence-base for the development of new innovative eHealth interventions for smoking cessation.

\section{Additional file}

Additional file 1: Table S1. Items from the World Health Organisation

Trial Registration Data Set as per SPIRIT guidelines. (DOCX 15 kb)

\section{Abbreviations}

AlH: Automatic Inhibition Hypothesis; ANOVA: Analysis of Variance; AUDIT: Alcohol Use Disorders Identification Test; BIS-11: Barratt Impulsiveness Scale; BSI: Behaviour Stimulus Interaction; CONSORT: Consolidated Standards of Reporting Trials; DASS21: Depression, Anxiety and Stress Scale; DSM-5: Diagnostic and Statistical Manual of Mental Disorders, Fifth Edition; DUHREC: The Deakin University Human Research Ethics Committee; FTND: Fagerström Test for Nicotine Dependence; GNG: Go/No-go; ID: Identification; INST: Inhibition Smoking Training; NRT: Nicotine Replacement Therapy; RCT: Randomised Controlled Trial; SSD: Stop Signal Delay; SSRT: Stop Signal Reaction Time; SST: Stop Signal Task; T1: Time 1 (baseline assessment); T2: Time 2 (post-intervention); T3: Time 3 (one month follow-up); T4: Time 4 (three months follow-up); TLFB: Timeline Follow-Back

\section{Acknowledgements}

The authors would like to thank the following people for their expertise and input: Ron Borland, Robert Dvorak, Frederick Verbruggen, Peter Enticott, Denise Foley, Sasha Davies, Ben Richardson, and Adrian Shatte. Thank you to the participants for participating and providing the feedback on this program.

\section{Funding}

This research is wholly funded by Deakin University. This funding source had no role in the design of the study and collection, analysis, interpretation of data or writing of the manuscript.

\section{Availability of data and materials}

The syntax and full analysis plan will be made available on the Open Science Framework and the dataset made available by contacting the first author. 


\section{Consent for image reproduction}

Pexels: Untitled image uploaded 18 November 2016. Accessed from Pixabay under Creative Commons CCO. Studio Art: Close-up view of male hand take a pack of cigarettes (Royalty-free stock photo ID: 281293961). Shutterstock; Accessed with permission 26 February 2016. Khamiranon D: Cigarettes With A Soft-Focus Background is Dark (Royalty-free stock photo ID:380941702). Shutterstock; Accessed with permission 26 February 2016.

\section{Authors' contributions}

PS is the project lead who has overall responsibility for the design and content of the intervention, and wrote the draft of the manuscript. MH is co-lead on this project and has shared responsibility for the design and content of the intervention and wrote the discussion section. KG is a Doctorate of Clinical Psychology candidate and led the piloting of the intervention. $\mathrm{LH}$ is a PhD student and along with JB, who is a Doctorate of Clinical Psychology candidate, contributed to the intervention design, the trial design, and will collect and analyse the data and write the first draft of the main papers. NL is the expert advisor on the response inhibition training intervention and provided all aspects of the control intervention and had input into the design. All authors contributed to the development of the protocol and writing of this paper, and have read and approved the final manuscript.

\section{Ethics approval and consent to participate}

Ethics for this study was approved by the Deakin University Human Research Ethics Committee (DUHREC) on 2nd February 2016 (Project ID: 2015-298). All participants provided written consent.

\section{Consent for publication}

Not applicable.

\section{Competing interests}

The authors declare that they have no competing interests.

\section{Publisher's Note}

Springer Nature remains neutral with regard to jurisdictional claims in published maps and institutional affiliations.

\section{Author details}

'School of Psychology, Deakin University, Geelong, VIC 3220, Australia. ${ }^{2}$ Centre for Drug Use, Addictive and Anti-social behaviour Research (CEDAAR), Deakin University, Locked Bag 20000, Geelong, VIC 3220, Australia. ${ }^{3}$ Cognitive Neuroscience Unit, School of Psychology, Deakin University, 221 Burwood Hwy, Burwood, VIC, Australia. ${ }^{4}$ Department of Psychology, University of Exeter, Perry Road, Prince of Wales Road, Exeter EX4 4QG, UK.

Received: 26 June 2018 Accepted: 11 October 2018 Published online: 03 November 2018

\section{References}

1. Forouzanfar $M H$, Alexander $L$, Anderson $H R$, Bachman VF, Biryukov $S$, Brauer $\mathrm{M}$, et al. Global, regional, and national comparative risk assessment of 79 behavioural, environmental and occupational, and metabolic risks or clusters of risks in 188 countries, 1990-2013: a systematic analysis for the global burden of disease study 2013. Lancet. 2015;386:2287-323.

2. World Health Organization [WHO]: WHO global report. Mortality attributable to tobacco. World Health Organization. 2012. http://www.who.int/tobacco/ publications/surveillance/rep_mortality_attributable/en/. Accessed 12 Oct 2017.

3. U.S. National Cancer Institute and World Health Organisation. The economics of tobacco and tobacco control. National Cancer Institute tobacco control monograph 21. Geneva: World Health Organization; 2016.

4. Australian Institute of Health and Welfare [AIHW]. National Drug Strategy Household Survey 2016: detailed findings. AlHW. 2017. https:/www.aihw. gov.au/getmedia/15db8c15-7062-4cde-bfa4-3c2079f30af3/21028.pdf. aspx?inline=true. Accessed 15 Oct 2017

5. Greenhalgh EM, Bayly M, Winstanley MH. Chapter 1. Trends in the prevalence of smoking. In: Scollo MM, Winstanley MH, editors. Tobacco in Australia: facts and issues. Melbourne: Cancer Council Victoria; 2015.

6. Stead LF, Lancaster T. Combined pharmacotherapy and behavioural interventions for smoking cessation. Cochrane Database Syst Rev. 2012;10:1-118.
7. Cahill K, Stevens S, Perera R, Lancaster T. Pharmacological interventions for smoking cessation: an overview and network meta-analysis. Cochrane Database Syst Rev. 2013;31:1-50.

8. Lancaster T, Stead LF. Individual behavioural counselling for smoking cessation. Cochrane Database Syst Rev. 2005;18:1-50.

9. Stead LF, Lancaster T. Group behaviour therapy programmes for smoking cessation. Cochrane Database Syst Rev. 2005;18:1-76.

10. Stead LF, Koilpillai P, Lancaster T. Additional behavioural support as an adjunct to pharmacotherapy for smoking cessation. Cochrane Database Syst Rev. 2015;10:1-104.

11. Centers for Disease Control and Prevention. Quitting smoking among adults-United States, 2000-2015. Morb Mortal Wkly Rep. 2017;65:1457-64.

12. Balmford J, Borland R, Hammond D, Cummings KM. Adherence to and reasons for premature discontinuation from stop-smoking medications: data from the ITC four-country survey. Nicotine Tob Res. 2011;13:94-102.

13. Morphett K, Partridge B, Gartner C, Carter A, Hall W. Why don't smokers want help to quit? A qualitative study of smokers' attitudes towards assisted vs. unassisted quitting. Int J Environ Res Public Health. 2015;12:6591-607.

14. Vogt F, Hall S, Marteau TM. Understanding why smokers do not want to use nicotine dependence medications to stop smoking: qualitative and quantitative studies. Nicotine Tob Res. 2008;10:1405-13.

15. Pisinger $C$, Aadahl $M$, Toft $U$, Jørgensen T. Motives to quit smoking and reasons to relapse differ by socioeconomic status. Prev Med. 2011;52:48-52.

16. Villanti AC, Manderski MTB, Gundersen DA, Steinberg MB, Delnevo CD. Reasons to quit and barriers to quitting smoking in US young adults. Fam Pract. 2016:33:133-9.

17. Hughes JR, Solomon LJ, Naud S, Fingar JR, Helzer JE, Callas PW. Natural history of attempts to stop smoking. Nicotine Tob Res. 2014;16:1190-8.

18. Piasecki TM. Relapse to smoking. Clin Psychol Rev. 2006;26:196-215.

19. Spanier CA, Shiffman S, Maurer A, Reynolds W, Quick D. Rebound following failure to quit smoking: the effects of attributions and self-efficacy. Exp Clin Psychopharm. 1996;4:191-7.

20. Krishnan-Sarin S, Reynolds B, Duhig AM, Smith A, Liss T, McFetridge A, et al. Behavioral impulsivity predicts treatment outcome in a smoking cessation program for adolescent smokers. Drug Alcohol Depend. 2007;88:79-82.

21. Powell J, Dawkins L, West R, Powell J, Pickering A. Relapse to smoking during unaided cessation: clinical, cognitive and motivational predictors. Psychopharmacology. 2010;212:537-49.

22. Charles-Walsh K, Furlong L, Munro DG, Hester R. Inhibitory control dysfunction in nicotine dependence and the influence of short-term abstinence. Drug Alcohol Depend. 2014;143:81-6.

23. Smith JL, Mattick RP, Jamadar SD, Iredale JM. Deficits in behavioural inhibition in substance abuse and addiction: a meta-analysis. Drug Alcohol Depend. 2014;145:1-33.

24. Yin J, Yuan K, Feng D, Cheng J, Li Y, Cai C, et al. Inhibition control impairments in adolescent smokers: electrophysiological evidence from a go/NoGo study. Brain Imaging Behav. 2016;10:497-505.

25. Billieux J, Gay P, Rochat L, Khazaal Y, Zullino D, Van der Linden M. Lack of inhibitory control predicts cigarette smoking dependence: evidence from a non-deprived sample of light to moderate smokers. Drug Alcohol Depend. 2010;112:164-7.

26. Venugopalan V. Compulsion and control: prefrontal and mesolimbic systems in human addiction (doctoral dissertation). Quebec: McGill University; 2011.

27. Hyland A, Borland R, Li Q, Yong HH, McNeill A, Fong GT, et al. Individual-level predictors of cessation behaviours among participants in the international tobacco control (ITC) four country survey. Tob Control. 2006;15:iiiz13.

28. Vangeli E, Stapleton J, Smit ES, Borland R, West R. Predictors of attempts to stop smoking and their success in adult general population samples: a systematic review. Addiction. 2011;106:2110-21.

29. Jones A, Di Lemma LC, Robinson E, Christiansen P, Nolan S, Tudur-Smith C, Field M. Inhibitory control training for appetitive behaviour change: a metaanalytic investigation of mechanisms of action and moderators of effectiveness. Appetite. 2016;97:16-28.

30. Jones A, McGrath E, Houben K, Nederkoorn C, Robinson E, Field M. A comparison of three types of web-based inhibition training for the reduction of alcohol consumption in problem drinkers: study protocol. BMC Public Health. 2014;14:796-805.

31. Wright L, Lipszyc J, Dupuis A, Thayapararajah S, Schachar R. Response inhibition and psychopathology: a meta-analysis of go/no-go task performance. J Abnorm Psychol. 2014;123:429-39. 
32. Verbruggen $F$, Logan GD. Response inhibition in the stop-signal paradigm. Trends Cogn Sci. 2008;12:418-24.

33. Allom V, Mullan B, Hagger M. Does inhibitory control training improve health behaviour? A meta-analysis. Health Psychol Rev. 2015;10:1-38.

34. Houben $\mathrm{K}$, Havermans RC, Nederkoorn C, Jansen A. Beer à no-go: learning to stop responding to alcohol cues reduces alcohol intake via reduced affective associations rather than increased response inhibition. Addiction. 2012;107:1280-7.

35. Jones $\mathrm{A}$, Field $\mathrm{M}$. The effects of cue-specific inhibition training on alcohol consumption in heavy social drinkers. Exp Clin Psychopharmacol. 2013;21:8-16.

36. Lawrence NS, O'Sullivan J, Parslow D, Javaid M, Adams RC, Chambers CD, et al. Training response inhibition to food is associated with weight loss and reduced energy intake. Appetite. 2015;95:17-28.

37. Veling $H$, van Koningsbruggen GM, Aarts $H$, Stroebe W. Targeting impulsive processes of eating behavior via the internet. Effects on body weight. Appetite. 2014;78:102-9.

38. Veling $\mathrm{H}$, Holland RW, van Knippenberg A. When approach motivation and behavioral inhibition collide: behavior regulation through stimulus devaluation. J Exp Soc Psychol. 2008;44:1013-9.

39. Houben K, Nederkoorn C, Wiers RW, Jansen A. Resisting temptation: decreasing alcohol-related affect and drinking behavior by training response inhibition. Drug Alcohol Depend. 2011;116(1-3).

40. Chen Z, Veling H, Dijksterhuis A, Holland RW. How does not responding to appetitive stimuli cause devaluation: evaluative conditioning or response inhibition? J Exp Psychol Gen. 2016;145:1687-701.

41. Veling $\mathrm{H}$, Aarts $\mathrm{H}$, Stroebe W. Stop signals decrease choices for palatable foods through decreased food evaluation. Front Psychol. 2013;4:1-7.

42. Veling $\mathrm{H}$, Aarts $\mathrm{H}$, Papies EK. Using stop signals to inhibit chronic dieters' responses toward palatable foods. Behav Res Ther. 2011;49:771-80.

43. Veling H, Lawrence $\mathrm{N}$, Chen Z, van Koningsbruggen GM, Holland RW. What is trained during food go/no-go training? A review focusing on mechanisms and a research agenda. Curr Addict Rep. 2017;4:35-41.

44. Pettiford J, Kozink RV, Lutz AM, Kollins SH, Rose JE, McClernon FJ. Increases in impulsivity following smoking abstinence are related to baseline nicotine intake and boredom susceptibility. Addict Behav. 2007;32:2351-7.

45. American Psychological Association [APA]. Diagnostic and statistical manual of mental disorders. 5th ed. Arlington: American Psychiatric Publishing; 2013.

46. Altman DG, Schulz KF. Concealing treatment allocation in randomised trials. BMJ. 2001;323:446-7.

47. Robinson SM, Sobell LC, Sobell MB, Leo Gl. Reliability of the timeline Followback for cocaine, cannabis, and cigarette use. Psychol Addict Behav. 2014;28:154-62.

48. Sobell LC, Sobell MB. Timeline follow-Back: a technique for assessing selfreported alcohol consumption. In: Litten RZ, Allen JP, editors. Measuring alcohol consumption: psychosocial and biochemical methods. Totowa: Humana Press; 1992. p. 41-72.

49. Heatherton TF, Kozlowski LT, Frecker RC, Fagerström KO. The Fagerström test for nicotine dependence: a revision of the Fagerström tolerance questionnaire. Br J Addict. 1991;86:1119-27.

50. Payne TJ, Smith PO, McCracken LM, McSherry WC, Antony MM. Assessing nicotine dependence: a comparison of the Fagerström tolerance questionnaire (FTQ) with the Fagerström test for nicotine dependence (FTND) in a clinical sample. Addict Behav. 1994;19:307-17.

51. Singleton EG, Anderson LM, Heishman SJ. Reliability and validity of the tobacco craving questionnaire and validation of a craving-induction procedure using multiple measures of craving and mood. Addiction. 2003;98:1537-46

52. West $\mathrm{R}$, Ussher M. Is the ten-item questionnaire of smoking urges (QSUbrief) more sensitive to abstinence than shorter craving measures? Psychopharmacology. 2010;208:427-32.

53. Roster CA, Lucianetti L, Albaum G. Exploring slider vs. categorical response formats in web-based surveys. J Res Pract. 2015;11:1-20.

54. Cook C, Heath F, Thompson R, Thompson B. Score reliability in web - or internet based surveys: unnumbered graphical rating scales versus Likerttype scales. Educ Psychol Meas. 2001;6:697-706.

55. Henry JD, Crawford JR. The short-form version of the depression anxiety stress scales (DASS-21): construct validity and normative data in a large non-clinical sample. Br J Clin Psychol. 2005;44:227-39.

56. Page AC, Hooke GR, Morrison DL. Psychometric properties of the depression anxiety stress scales (DASS) in depressed clinical samples. Br J Clin Psychol. 2007;46:283-97.
57. Saunders JB, Aasland OG, Babor TF, De La Fuente JR, Grant M. Development of the alcohol use disorders identification test (AUDIT): WHO collaborative project on early detection of persons with harmful alcohol consumption-ll. Addiction. 1993:88:791-804.

58. Reinert DF, Allen JP. The alcohol use disorders identification test: an update of research findings. Alcohol Clin Exp Res. 2007:31:185-99.

59. Patton JH, Stanford MS, Barratt ES. Factor structure of the Barratt impulsiveness scale. J Clin Psychol. 1995;51:768-74.

60. Spinella M. Normative data and a short form of the Barratt impulsiveness scale. Int J Neurosci. 2007;117:359-68.

61. Lappin JS, Eriksen CW. Use of a delayed signal to stop a visual reaction-time response. J Exp Psychol Gen. 1966;72:805-11.

62. Logan GD, Schachar RJ, Tannock R. Impulsivity and inhibitory control. Psychol Sci. 1997;8:60-4.

63. Houben $\mathrm{K}$, Nederkoorn C, Jansen A. Eating on impulse: the relation between overweight and food-specific inhibitory control. Obesity. 2014;22:E6-8.

64. Field A. Discovering statistics using IBM SPSS statistics. 5th revised ed. London: Sage Publications Ltd; 2013.

65. Allison PD. Multiple imputation for missing data: a cautionary tale. Sociol Methods Res. 2000;28:301-9.

66. Horton NJ, Lipsitz SR. Multiple imputation in practice: comparison of software packages for regression models with missing variables. Am Stat. 2001;55:244-54.

67. Adams S, Mokrysz C, Attwood AS, Munafo MR. Resisting the urge to smoke: inhibitory control training in cigarette smokers. R Soc Open Sci. 2017;4: 170045.

68. Stice E, Yokum S, Veling H, Kemps E, Lawrence NS. Pilot test of a novel food response and attention training treatment for obesity: brain imaging data suggest actions shape valuation. Behav Res Ther. 2017;94:60-70.

69. Adams RC, Lawrence NS, Verbruggen F, Chambers CD. Training response inhibition to reduce food consumption:mechanisms, stimulus specificity and appropriate training protocols. Appetite. 2016;109:11-23.

70. Abroms LC, Lee Westmaas J, Bontemps-Jones J, Ramani R, Mellerson J. A content analysis of popular smartphone apps for smoking cessation. Am J Prev Med. 2013;45:732-6.

71. Pexels: Untitled Image from Pixabay [Internet]. Uploaded 18 November 2016. Available from .https://pixabay.com/en/lake-forest-woods-personbench-2611710/.

72. Studio Art: Close-up view of male hand take a pack of cigarettes [Internet]. Available from https://www.shutterstock.com/image-photo/closeup-viewmale-hand-take-pack-281293961. Accessed 26 Feb 2016.

73. Khamiranon D: Cigarettes With A Soft-Focus Background Is Dark [Internet]. Accessed 26 Feb 2016. Available from https://www.shutterstock.com/imagephoto/cigarettes-softfocus-background-dark-380941702
Ready to submit your research? Choose BMC and benefit from:
- fast, convenient online submission
- thorough peer review by experienced researchers in your field
- rapid publication on acceptance
- support for research data, including large and complex data types
- gold Open Access which fosters wider collaboration and increased citations
- maximum visibility for your research: over $100 \mathrm{M}$ website views per year
At BMC, research is always in progress.
Learn more biomedcentral.com/submissions 